\title{
Direct probing of ion pair formation using a symmetric triangulenium dye
}

Westerlund, Axel Rune Fredrik; Elm, Jonas; Jørgensen, Jacob Lykkebo; Carlsson, Nils; Thyrhaug, Erling; Åkerman, Björn; Sørensen, Thomas Just; Mikkelsen, Kurt Valentin; Laursen, Bo Wegge

Published in:

Photochemical \& Photobiological Sciences

Publication date:

2011

Document version

Publisher's PDF, also known as Version of record

Citation for published version (APA):

Westerlund, A. R. F., Elm, J., Jørgensen, J. L., Carlsson, N., Thyrhaug, E., Åkerman, B., Sørensen, T. J.,

Mikkelsen, K. V., \& Laursen, B. W. (2011). Direct probing of ion pair formation using a symmetric triangulenium dye. Photochemical \& Photobiological Sciences, 10, 1963-1973. 
Cite this: Photochem. Photobiol. Sci., 2011, 10,1963

\title{
Direct probing of ion pair formation using a symmetric triangulenium dye $\dagger$
}

\author{
Fredrik Westerlund, ${ }^{a, b}$ Jonas Elm, ${ }^{a}$ Jacob Lykkebo, ${ }^{a}$ Nils Carlsson, ${ }^{b}$ Erling Thyrhaug, ${ }^{a}$ Björn Åkerman, ${ }^{b}$ \\ Thomas Just Sørensen, ${ }^{a}{ }^{K u r t ~ V . ~ M i k k e l s e n ~}{ }^{a}$ and Bo W. Laursen ${ }^{* a}$
}

\author{
Received 16th August 2011, Accepted 14th September 2011 \\ DOI: 10.1039/c1pp05253e
}

The 2,6,10-tris(dialkylamino)trioxatriangulenium dyes (ATOTA ${ }^{+}$) are highly stabilised cationic chromophores with $D_{3 \mathrm{~h}}$ symmetry. The symmetry gives rise to a degeneracy of the main electronic transition. In low polarity solvents significant splitting of this degenerate transition is observed and assigned to ion pair formation. Ion pairing of the 2,6,10-tris(dioctylamino)trioxatriangulenium ion with $\mathrm{Cl}^{-}, \mathrm{BF}_{4}^{-}, \mathrm{PF}_{6}{ }^{-}$and TRISPHAT anions was studied using absorption spectroscopy. A clear correlation is found between the size of the anion and the splitting of the ATOTA ${ }^{+}$transitions. In benzene the $\mathrm{Cl}^{-}$salt displays a splitting of $1955 \mathrm{~cm}^{-1}$, while the salt of the much larger TRISPHAT ion has a splitting of $1543 \mathrm{~cm}^{-1}$. TD-DFT calculations confirm the splitting of the states and provide a detailed insight into the electronic structure of the ion pairs. The different degree of splitting in different ion pairs is found to correlate with the magnitude of the electric field generated in each ion pair, thus leading to the conclusion that the effect seen is an internal Stark effect. By insertion of an amphiphilic derivative of the $\mathrm{ATOTA}^{+}$chromophore in an oriented lamellar liquid crystal, it was possible to resolve the two bands of the double peak spectrum and show their perpendicular orientation in the molecular framework, as predicted by the calculations.

\section{Introduction}

Cationic dyes, such as cyanines, rhodamines, and triphenylmethyliums (TPMs), are used in a wide variety of applications, including bioimaging, ${ }^{1-5}$ sensing, ${ }^{6-9}$ and material science. ${ }^{10-16}$ The fact that these dyes are cationic implies that they are associated with a counterion. Interactions between the dye and surrounding anions may affect the photophysical properties, ${ }^{17-20}$ but such effects are in most cases not easily quantified, as they are hard to separate from other effects. Thus, a simple shift in wavelength of absorption upon change in solvent composition may originate from a purely solvatochromic effect, specific interactions with the counterion, or from non-specific electrostatic interactions between the two ions. Spectral shifts resulting from electric fields are well known from Stark spectroscopy. ${ }^{21}$ Similarly, voltage sensitive dyes are important tools in molecular biology to probe changes in membrane potential. ${ }^{6,22}$

In dyes with high symmetry such as TPMs the $\mathrm{S}_{1}$-transition is degenerate. ${ }^{19}$ If the dye and counterion come into close contact

aDepartment of Chemistry \& Nano-Science Center, University of Copenhagen, Universitetsparken 5, DK-2100, København Ø, Denmark. E-mail: bwl@nano.ku.dk; Fax: +45 3532 0214; Tel: +45 35321881

${ }^{b}$ Department of Chemical and Biological Engineering, Chalmers University of Technology, Kemivägen 10, SE-41296, Göteborg, Sweden

$\dagger$ Electronic supplementary information (ESI) available: Optimized geometries for ATOTA.X, calculated vacuum excitation energies, calculated excitation energies for the ion pair geometry of $\mathrm{ATOTA}^{+}$, calculated transition dipole moments, absorption titrations and additional spectroscopy. See DOI: $10.1039 / \mathrm{cl} p$ p05253e and are asymmetrically positioned, the degenerate $S_{1}$-transition will split due to the electric field of the anion, resulting in two absorption peaks. This phenomenon has been reported for TPMs like crystal violet and ethyl violet. ${ }^{23-28}$ However, quantifying this effect is complicated due to the conformational flexibility and non-fluorescent nature of the TPM dyes. It has thus been an ongoing discussion if the observed symmetry breaking in these dyes originates from conformational changes, specific interactions with nucleophiles, or purely electrostatic effects.

We have previously introduced dyes based on the 2,6,10tris(dialkylamino)trioxatriangulenium ion $\left(\right.$ ATOTA $^{+}$, Fig. 1) ${ }^{17,29}$ These dyes may be considered as crystal violet analogues with all three phenyl rings locked into the same plane by ortho oxygen bridges. The rigid triangulenium ring system efficiently blocks the dominating modes of non-radiative deactivation in TPM dyes and makes ATOTA $^{+}$and other triangulenium dyes efficient fluorophores. ${ }^{13,30-38}$ The triangulenium ring system furthermore provides very efficient delocalisation of the positive charge, resulting in extremely stabilised cations that are particularly insensitive to nucleophiles. ${ }^{29,37,38}$

In a previous study of the photophysical properties of $\mathrm{ATOTA}^{+}$, we identified three distinct interaction states of the dye and its $\mathrm{PF}_{6}{ }^{-}$ counterion. ${ }^{17}$ In polar solvents (here dichloromethane (DCM)) the dye is well separated from its anion and displays a single absorption band from the doubly degenerate transition. In benzene and other apolar, but polarisable, solvents an ion pair is formed. The ion pair spectrum displays two clear peaks assigned to originate from symmetry breaking. In apolar and non-polarisable solvents (here 


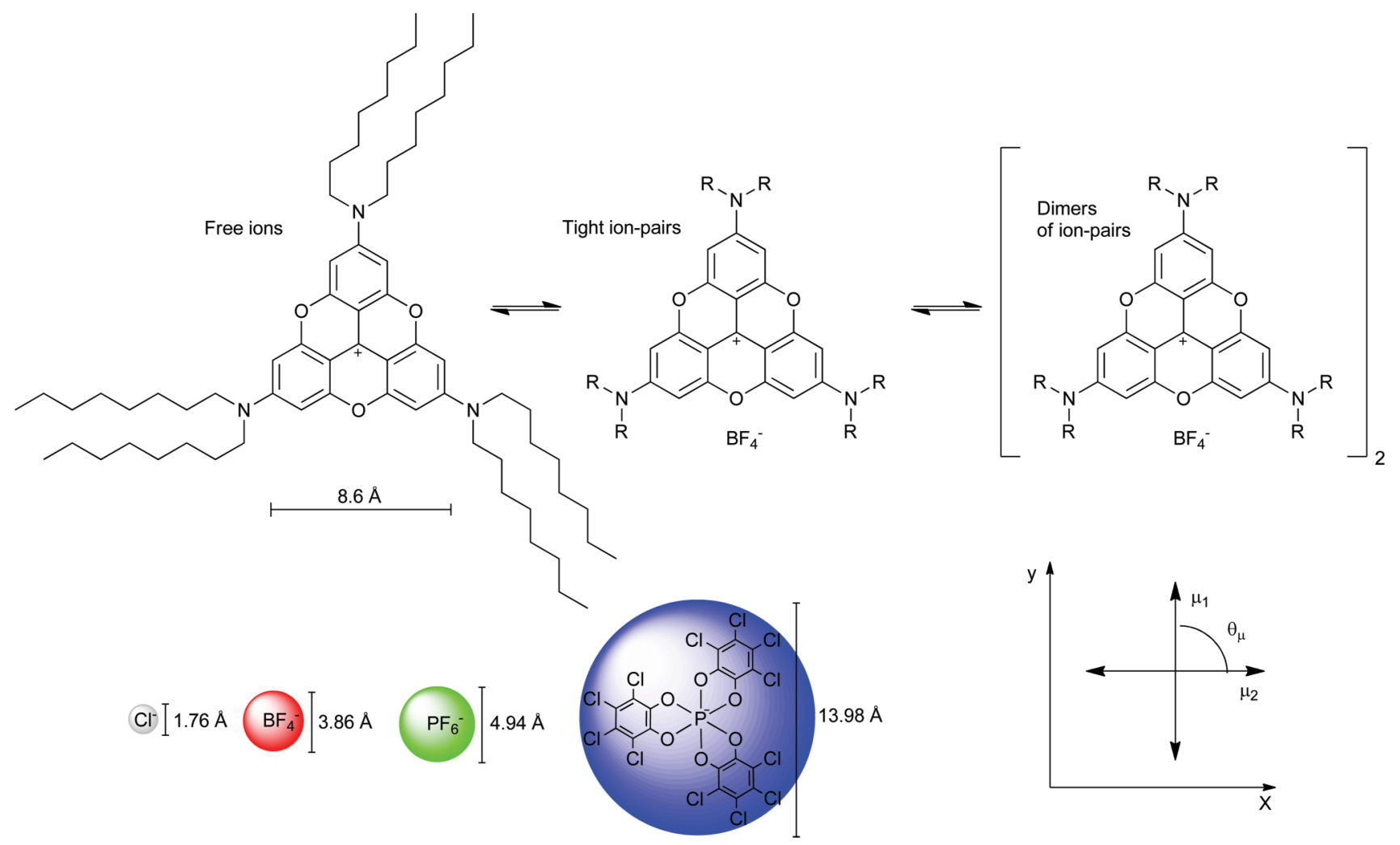

Fig. 1 Top: Schematic presentations of the different states of association of 2,6,10-tris(dioctylamino)-trioxatriangulenium $\left(\right.$ ATOTA $\left.^{+}\right)$and its counterion (here exemplified by $\mathrm{BF}_{4}^{-}$): free ion, ion pair and dimer of ion pairs. The width of the ATOTA ${ }^{+}$core is also indicated. Bottom left: The molecular structure and size of chloride (grey), tetrafluoroborate (red), hexafluorophosphate (green) and TRISPHAT (TT- ${ }^{-}$, blue). Bottom right: The coodinate system used for discussion of the transition moments $\mu_{1}$ and $\mu_{2}$ and their mutual angle $\theta$.

methylcyclohexane $(\mathrm{MCH})$ ), a dimer of ion pairs is formed. The dimer formation is revealed by absorption and fluorescence typical for an $\mathrm{H}$-aggregate. Based on these findings we expected that the $\mathrm{ATOTA}^{+}$dyes could be effective probes for studying ion pairing in detail. Firstly, the spectral properties of the ATOTA ${ }^{+}$ion are highly sensitive to ion pair formation. Secondly, the rigidity and high cation stability of the ATOTA ${ }^{+}$ion makes it unlikely that the observed effects are influenced by specific interactions with nucleophiles and conformational changes of the dye.

Here, we present a combined spectroscopic and computational study of the structure and photophysical properties of a series of ATOTA $^{+}$ion pairs. The study was designed to provide a detailed model of the electric field experienced by the ATOTA ${ }^{+}$ chromophore in tight ion pairs. Four salts of the symmetric noctyl substituted ATOTA ${ }^{+}$cation (2,6,10-tris(di-n-octylamino)trioxatriangulenium, $\mathbf{1}, \mathrm{ATOTA}^{+}$) and anions of varying size were studied to provide a correlation between the electric field and the splitting of the electronic states in the intrinsically symmetric chromophore. We demonstrate that there is a significant size effect on the splitting that can be assigned to an internal Stark effect.

\section{Experimental}

\section{Materials}

The synthesis of 2,6,10-tris(dioctylamino)trioxatriangulenium hexafluorophosphate (ATOTA $\cdot \mathrm{PF}_{6}$ ) is described in reference 29. The $\mathrm{Cl}^{-}$salt was prepared from the $\mathrm{PF}_{6}{ }^{-}$salt by ion exchange using

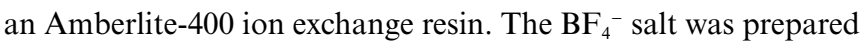
from the $\mathrm{Cl}^{-}$salt by repeated precipitation with $0.2 \mathrm{M} \mathrm{NaBF}_{4}$ (aq) from acetonitrile. The $\mathrm{TT}^{-}$salt was prepared by addition of cinchonidine $\Delta-\mathrm{TT}^{39}$ to ATOTA.PF $\mathrm{F}_{6}$ followed by column chromatography. The ion exchange was verified by ${ }^{19} \mathrm{~F}$ and ${ }^{31} \mathrm{P}$ NMR.

\section{Spectroscopy}

The absorption measurements were performed using a standard UV/vis spectrometer. The titration series with changing solvent composition were performed by diluting with one of the solvents and subsequently correcting the absorption spectra for dilution. Spectra with absorptions that appear to be above 2 in the figures were thus in reality recorded at lower optical density. The titration series were analysed by projecting the spectra in the corresponding pure solvents on all spectra in the titration series and the contribution of each of the two pure spectra is plotted in the graphs. The spectrum of the ATOTA.TT ion pair in benzene was calculated by comparing the measured spectrum in benzene with the spectra in the titration series when DCM was added to $\mathrm{MCH}$. The spectrum at $13 \mathrm{v} / \mathrm{v} \% \mathrm{DCM}$ is similar in shape and the analysis discussed above reveals that the spectrum of ATOTA.TT in benzene has a component of the spectrum of free ATOTA ${ }^{+}$ corresponding to $25 \%$ of the total spectrum. The spectrum of the ion pair alone in benzene was then calculated by subtracting the corresponding amount of the spectrum of free $\mathrm{ATOTA}^{+}$from the measured spectrum in benzene. 


\section{Calculations}

All molecular structures were optimised in Gaussian 03. The geometry optimisations were done with Beckes(B88) 3-parameter Exchange potential ${ }^{40}$ combined with the correlation energy of Lee, Yang and Parr (LYP $)^{41}$ into the well-known hybrid functional B3LYP $^{42,43}$ All excitation energies were calculated using the new coulomb attenuating method CAM-B3LYP. ${ }^{44}$ It has been shown that B3LYP is inadequate to calculate charge transfer excitations. ${ }^{45}$ This inadequacy is due to the asymptotic limit in the B88 exchange potential which goes off at $0.2 / r$ instead of the exact value of $1 / r{ }^{46}$ This is corrected with the coulomb attenuating method by including more exact HF exchange at long range. CAM-B3LYP has previously been shown to perform well in charge transfer excitations. ${ }^{47}$ All excitation energies were calculated in DALTON ${ }^{48}$ using the CAM-B3LYP functional. See ESI for details on used basis sets and convergence of basis set. $\dagger$

All structure optimisations where performed on the full ATOTA $^{+}$cation including the six n-octyl chains. Before calculations of excitation energies the side chains were substituted by ethyl groups. The $\mathrm{TT}^{-}$anions is chiral, ${ }^{39,49}$ and the $\Delta$ isomer was used in all calculations. For excitation calculations including a continuum solvent model a cavity of $18 \mathrm{Bohr}(\approx 9.5 \AA)$ was applied in all cases except for the $\mathrm{TT}^{-}$ion pair where a cavity of $24 \operatorname{Bohr}(\approx 12.7 \AA)$ was used.

\section{Lamellar liquid crystal}

The lamellar liquid crystal system was prepared by mixing $8.2 \%$ sodium octanoate (Sigma), 23.2\% decanol (L. Light \& Co) and 68.6\% MQ water, by weight. 2-didecylamino-6,10bis(dimethylamino)-4,8,12-trioxatriangulenium hexafluorophosphate $\left(\mathbf{2} \cdot \mathrm{PF}_{6}\right)$ was synthesised as described elsewhere. ${ }^{16} \mathrm{~A}$ chloroform solution of $2 \cdot \mathrm{PF}_{6}(5 \mu \mathrm{l}, 10 \mathrm{mM})$ was added to the $\mathrm{LC}$ solution $(150 \mu \mathrm{l})$ in the preparation step. The samples were left at room temperature in the dark to equilibrate for at least one week. The resulting phase is composed of stacked $2.5 \mathrm{~nm}$ thick octanoate/decanol double layers separated by $5.0 \mathrm{~nm}$ thick water layers. ${ }^{50,51}$ The samples were placed between two quartz plates with a $0.1 \mathrm{~mm}$ spacer chiseled in one of them. In order to increase the macroscopic orientation of the bilayers parallel to the quartz plates, the samples were heated to $100{ }^{\circ} \mathrm{C}$ and then cooled to room temperature. ${ }^{52}$ Linear dichroism spectra were measured on a CD spectrometer fitted with a linear dichroism detector with the plates mounted in a goniometer cell holder ${ }^{53}$ adjusting the grazing angle to $60^{\circ}$. A spectrum recorded on a sample without $2^{+}$at the same grazing angle was used as a baseline. Absorption spectra were recorded on the same anisotropic samples at a $90^{\circ}$ grazing angle and baseline corrected in the same manner.

The absorption spectrum presented in Fig. 10 is measured on an oriented sample, so the measured spectrum $\left(A_{\mathrm{m}}\right)$ is not equal to the isotropic absorption $\left(A_{\text {iso }}\right)$. $A_{\text {iso }}$ can be calculated using:

$$
A_{\text {iso }}=A_{\mathrm{m}}+\mathrm{LD}^{\omega=0} / 3
$$

where $\omega$ is the grazing angle and $A_{\mathrm{m}}$ is the measured absorbance. The measured $\mathrm{LD}^{\omega=60}$ was extrapolated to $\mathrm{LD}^{\omega=0}$ according to the procedure presented by Nordén et al. ${ }^{52}$ The calculated $A_{\text {iso }}$ is plotted in Fig. 10 and compared to $A_{\mathrm{m}}$ in Fig. S7 in the ESI. $\dagger$

\section{Results and discussion}

2,6,10-Tris(di-n-octylamino)trioxatriangulenium (ATOTA ${ }^{+}$) was used as the cation/probe to ensure good solubility in a wide range of low polarity solvents. Chloride $\left(\mathrm{Cl}^{-}\right)$, tetrafluoroborate $\left(\mathrm{BF}_{4}{ }^{-}\right)$, hexafluorophosphate $\left(\mathrm{PF}_{6}{ }^{-}\right)$and $\Delta$-tris(tetrachloro-1,2benzenediolato)phosphate(v) ${ }^{39}$ (TRISPHAT, TT $^{-}$) were selected as anions. The structures and relative sizes of the ions are illustrated in Fig. 1. The free $\mathrm{ATOTA}^{+}$cation is characterised by a single sharp absorption band at $476 \mathrm{~nm}$ (DCM) and a small Stokes' shift. ${ }^{29}$ The absorption spectra of $\mathrm{ATOTA}^{+}$in DCM are identical for all four anions investigated (see ESI, Fig. $\mathrm{S} 1 \dagger$ ), and identical to the spectra presented in our first study, ${ }^{17}$ confirming that the dye is unaffected by the nature of the counterion in polar solvents. Thus, the four starting materials have identical photophysical characteristics and any observed differences are related to interactions between $\mathrm{ATOTA}^{+}$and the respective counterion.

The model proposed in our previous study stated that the ATOTA $^{+}$dye can exist in three different states (Fig. 1), free dye, ion pair and a dimer of ion pairs, depending on the polarity of the solvent. ${ }^{17}$ Each species has a distinct absorption spectrum, due to the symmetry imposed on the chromophore. The change is observable as the free ATOTA ${ }^{+}$chromophore has two degenerate electronic transitions illustrated by the transition dipole moments, $\mu_{1}$ and $\mu_{2}$ in Fig. 1. In the ion pair the single absorption band is split into two, presumably by the electric field induced by the anion. Upon formation of dimers of ion pairs, two chromophores interact, which gives a broad absorption resulting from exciton coupling between the two. ${ }^{17}$ Fig. 1 shows the equilibria between the three species. The positions of the equilibria are expected to be determined by the strength of the Coulomb forces between the ions, and hence depend on solvent polarity. The strength of this interaction is governed by the distance between the ions and hence the size of the counterion. The respective size of the ions is shown in Fig. 1, given as the diameter of a sphere formed by the largest calculated inter-atomic distance plus the vdW radius of the involved atoms. For $\mathrm{Cl}^{-}, \mathrm{BF}_{4}^{-}$and $\mathrm{PF}_{6}^{-}$this assumption is good, but for the flat $\mathrm{ATOTA}^{+}$cation and the propeller-shaped $\mathrm{TT}^{-}$anion it only gives the largest dimension.

Before detailed comparative studies of the various ion pairs could be performed it was necessary to establish the experimental window for these species. Thus, conditions for formation of ion pairs and dimers of ion pairs were studied systematically for the four different ATOTA $^{+}$salts. The handle to control the Coulomb forces is the polarity of the solvent, as the interaction energy scales with the inverse of the relative dielectric constant of the medium $\left(\varepsilon_{\mathrm{r}}\right)$. Fig. $2 \mathrm{~A}$ shows a titration of ATOTA $\cdot \mathrm{BF}_{4}$ in benzene with increasing concentration of DCM. In pure benzene the dye exists exclusively as a tight ion pair, ${ }^{17}$ which is confirmed by measurements at different concentrations (ESI, Fig. S2 $\dagger$ ). In the titration with DCM there is a gradual transition from the characteristic double peak spectrum of the ion pair to the spectrum of the free dye when the volume fraction of DCM increases. This equilibrium may involve several species and go from contact ion pair, over solvent shared ion pair, and solvent separated ion pair, to the free ions. ${ }^{54}$ Such intermediate ion pair species and solvatochromic effects explain why the two peaks from the ion pair gradually merge into the single peak of the free ion, and why 

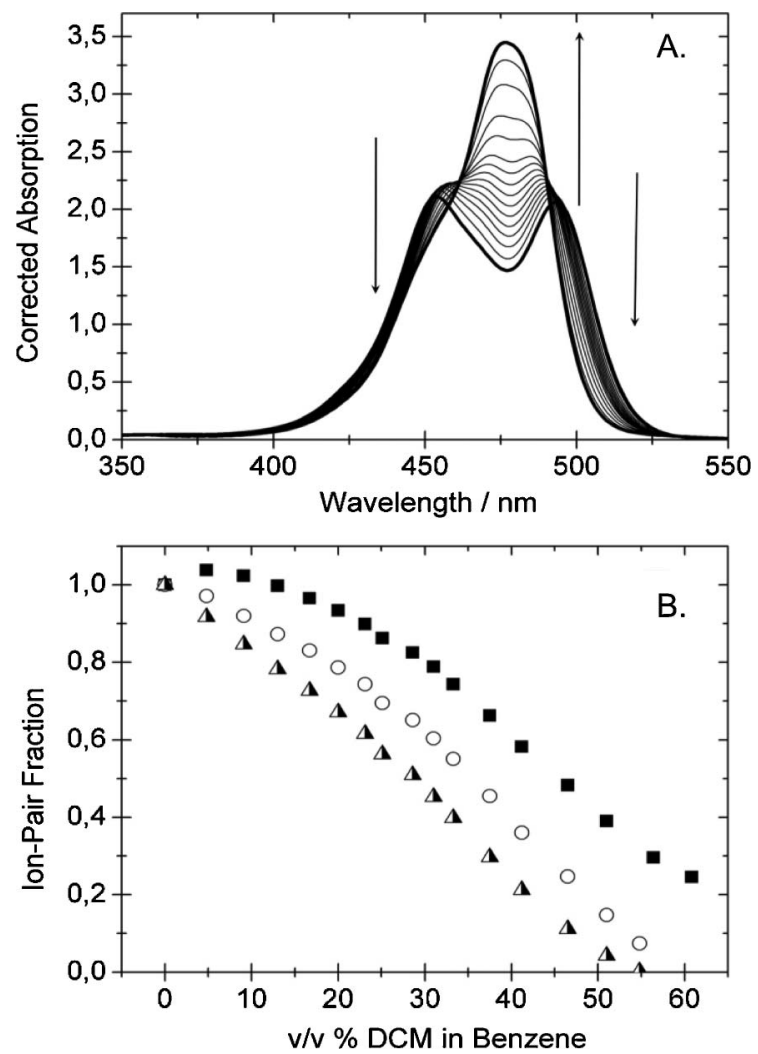

Fig. 2 A. Titration of ATOTA $\cdot \mathrm{BF}_{4}$ in benzene with increasing volume fraction DCM. The titration starts in pure benzene and ends in approximately $60 \mathrm{v} / \mathrm{v} \% \mathrm{DCM}$. The two extreme spectra are shown in bold. The spectra are corrected for dilution. B. Ion pair fraction of the absorption spectrum as a function of the volume percent DCM added, with $\mathrm{Cl}^{-}(\mathbf{\square})$, $\mathrm{BF}_{4}^{-}(\bigcirc)$ or $\mathrm{PF}_{6}^{-}(\boldsymbol{\Delta})$ as counterion.

isosbestic points are not observed for the whole data set. Similar titrations were performed for the $\mathrm{Cl}^{-}$and $\mathrm{PF}_{6}{ }^{-}$salts, showing the same trend (ESI, Fig. S3†). To analyse the effect of the counterion on the equilibrium between free dye and ion pair, the spectra in pure benzene and DCM were projected on all the spectra of the titrations (see Experimental section for details). The ion pair fraction of each spectrum is plotted as a function of the volume percent DCM for the different counterions in Fig. 2B. The salt with the smallest anion clearly forms the strongest ion pairs, and the strength decreases with size. The trace for the $\mathrm{Cl}^{-}$salt reaches a value slightly higher than one at low DCM concentrations. This can be explained by a small fraction of the $\mathrm{Cl}^{-}$ion pairs forming dimers in pure benzene at the concentration where the titration is performed (approximately $1 \times 10^{5} \mathrm{M}^{-1}$ ). This is in agreement with spectra recorded at different concentrations in pure benzene (ESI, Fig. S2 $\dagger$ ) where the $\mathrm{Cl}^{-}$salt shows the most pronounced concentration dependence.

To investigate the equilibrium between ion pairs and their dimers, titrations with benzene in $\mathrm{MCH}$ were performed. The spectra for ATOTA $\cdot \mathrm{BF}_{4}$ are shown in Fig. $3 \mathrm{~A}$ with a clear change observed when going from dimer to ion pair as the benzene concentration increases. The titration supports a simple two component equilibrium between monomeric and dimeric ion pairs as previously reported for ATOTA. $\mathrm{PF}_{6}$ when varying concentration and temperature. ${ }^{17}$ The minor sliding of the isosbestic points are
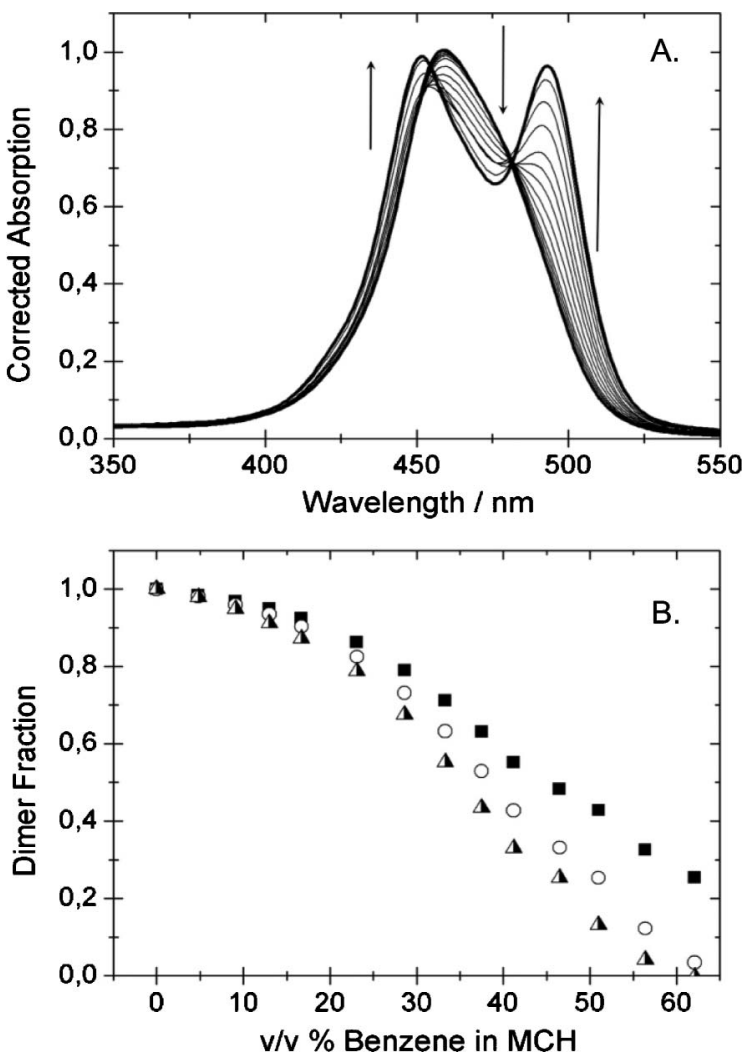

Fig. 3 A. Titration of ATOTA $\cdot \mathrm{BF}_{4}$ in $\mathrm{MCH}$ with increasing volume fraction benzene. The titration starts in pure $\mathrm{MCH}$ and ends in approximately $60 \mathrm{v} / \mathrm{v} \%$ benzene. The two extreme spectra are shown in bold. The spectra are corrected for dilution. B. Ion pair fraction of the absorption spectrum as a function of the volume percent benzene with $\mathrm{Cl}^{-}(\mathbf{\square}), \mathrm{BF}_{4}^{-}(\mathrm{O})$ or $\mathrm{PF}_{6}{ }^{-}(\boldsymbol{\Delta})$ as counterion.

again attributed to solvatochromic effects in the non-constant dielectric medium. Similar results are obtained in $\mathrm{MCH} /$ benzene titrations of the $\mathrm{Cl}^{-}$and $\mathrm{PF}_{6}{ }^{-}$salts (ESI, Fig. S4 $\dagger$ ). The relative fractions of ion pairs and their dimers were analysed as described above (Fig. 3B). Dimers formed by the $\mathrm{Cl}^{-}$salt are the most strongly associated and require the highest benzene fraction to dissociate. This is in good agreement with the observation that dimers of the $\mathrm{Cl}^{-}$salt are formed to some extent in pure benzene at elevated concentrations (ESI, Fig. S2 $\dagger$ ).

Finally, a titration with DCM from $\mathrm{MCH}$ was performed to investigate to which extent the tight ion pair is present when going from the dimer all the way to the free ions. Fig. 4 shows the titration for ATOTA $\mathrm{BF}_{4}^{-}$(the corresponding titrations for the $\mathrm{Cl}^{-}$and $\mathrm{PF}_{6}{ }^{-}$salts are given in the ESI, Fig. $\left.\mathrm{S} 5 \dagger\right)$. The dimers of ion pairs are solvated, first into ion pairs, and subsequently into free ions when the volume fraction of DCM increases. The characteristic double peak of the ion pair is clearly seen during the titration, yet in this solvent system the free cation starts to form before the second equilibrium (Fig. 1) is shifted completely into the tight ion pair.

The conclusions from the titration series for the three smallest ions are: (i) $\mathrm{The}^{-} \mathrm{Cl}^{-}$salt has the strongest tendency to form ion pairs and ion pair dimers, a tendency that decreases with increasing size of the anion. (ii) At low concentrations (below $1 \times 10^{-6} \mathrm{M}$ ) in pure benzene solution, the ATOTA ${ }^{+}$salts of $\mathrm{Cl}^{-}, \mathrm{BF}_{4}^{-}$and 


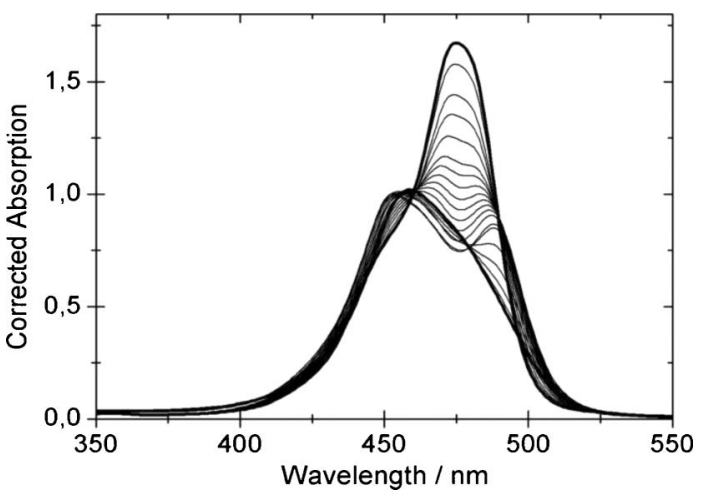

Fig. 4 Titration of ATOTA $\cdot \mathrm{BF}_{4}$ in $\mathrm{MCH}$ with increasing volume fraction DCM. The titration starts in pure $\mathrm{MCH}$ and ends in approximately $55 \mathrm{v} / \mathrm{v}$ $\%$ DCM. The two extreme spectra are shown in bold. The spectra are corrected for dilution.

$\mathrm{PF}_{6}{ }^{-}$are present almost exclusively as tight ion pairs, which allows us to directly compare the spectral effects induced by the different counterions. (iii) The behaviour of the $\mathrm{TT}^{-}$salt differs significantly from the three other anions, especially in low polarity solvents. The latter will be discussed in detail below.

The absorption spectrum of ATOTA $^{+}$in benzene changes significantly with the four different counterions (Fig. 5) and there are two main observations to be made. First, the splitting of the two peaks increases with decreasing size of the counterion (Table 1). This supports our hypothesis that a smaller counterion will be able to get closer to the dye and expose the dye to a larger electric field, causing a larger splitting. Second, the intensity of the $S_{2}$-transition decreases relative to $S_{1}$ with increasing size of the counterion. The spectra are relatively insensitive to concentration (see ESI, Fig. S2 $\dagger$ ) with ATOTA.Cl showing some aggregation at elevated concentrations, vide supra.

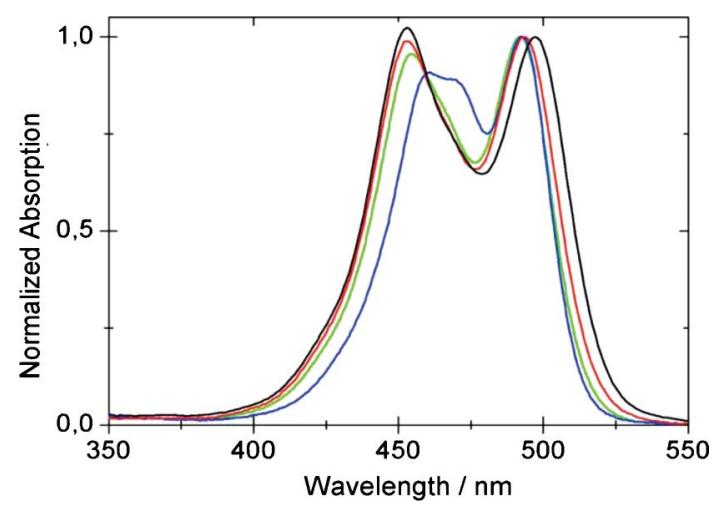

Fig. 5 Normalized absorption spectra of ATOTA $^{+}$in benzene with different counterions: $\mathrm{Cl}^{-}$(black), $\mathrm{BF}_{4}^{-}$(red), $\mathrm{PF}_{6}{ }^{-}$(green) and $\mathrm{TT}^{-}$(blue). Concentration: $5 \times 10^{-6} \mathrm{M}$. Spectra normalised at the $\mathrm{S}_{1}$ transition.

In benzene (Fig. 5), ATOTA.TT displays an extra peak at 470 $\mathrm{nm}$ between the two main peaks. In MCH solution, on the other hand, where the three other salts are fully dimerised, ATOTA.TT displays a spectrum with two peaks, very similar to the spectra of the other ion pairs in benzene (Fig. 6). This indicates that ATOTA.TT does not form dimers in MCH in the concentration range studied, but exists only as ion pairs, most likely due to the large size of the $\mathrm{TT}^{-}$anion. Titration of $\mathrm{MCH}$ and benzene
Table 1 Experimental splitting of the ion pair peaks

\begin{tabular}{|c|c|c|c|c|c|}
\hline \multirow[b]{2}{*}{ Counterion (solvent) } & \multicolumn{2}{|l|}{$\mathrm{S}_{1}$} & \multicolumn{2}{|l|}{$\mathrm{S}_{2}$} & \multirow{2}{*}{$\frac{\mathrm{S}_{2}-\mathrm{S}_{1}}{\Delta E\left(\mathrm{~cm}^{-1}\right)}$} \\
\hline & $\lambda(\mathrm{nm})$ & $v\left(\mathrm{~cm}^{-1}\right)$ & $\lambda(\mathrm{nm})$ & $v\left(\mathrm{~cm}^{-1}\right)$ & \\
\hline $\mathrm{Cl}^{-}$(benzene) & 497 & 20120 & 453 & 22075 & 1955 \\
\hline $\mathrm{BF}_{4}^{-}$(benzene) & 493 & 20284 & 453 & 22075 & 1791 \\
\hline $\mathrm{PF}_{6}^{-}$(benzene) & 492 & 20325 & 454 & 22026 & 1701 \\
\hline$\Delta \mathrm{TT}^{-a}$ (benzene) & 493 & 20284 & 461 & 21692 & 1408 \\
\hline$\Delta \mathrm{TT}^{-b}$ (benzene) & 494 & 20243 & 459 & 21786 & 1543 \\
\hline$\Delta \mathrm{TT}^{-c}(\mathrm{MCH})$ & 491 & 20367 & 455 & 21978 & 1611 \\
\hline $\mathrm{LLC}^{d}$ & 488 & 20492 & 450 & 22222 & 1730 \\
\hline
\end{tabular}

${ }^{a}$ Peak splitting in the measured spectrum (benzene), ${ }^{b}$ Extrapolated splitting for the pure ion pair in benzene, ${ }^{c}$ Splitting in the measured spectrum in $\mathrm{MCH} .{ }^{d}$ Splitting in lamellar liquid crystal.

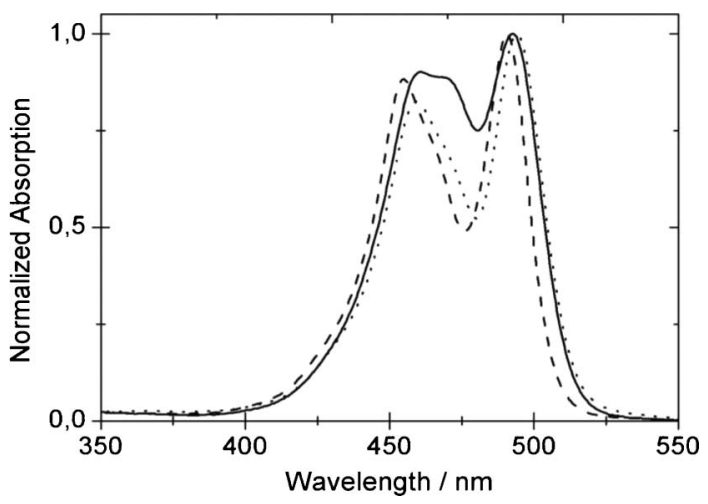

Fig. 6 Normalized absorption spectra of ATOTA.TT in benzene (solid line) and $\mathrm{MCH}$ (dashed line). The dotted line is the extrapolated spectrum for the pure ion pair in benzene (see Experimental section). Absorption spectra measured at $5 \times 10^{-6} \mathrm{M}$.

solutions of ATOTA.TT with DCM confirms this hypothesis (ESI, Fig. S6A-B $\dagger$ ). Furthermore, the spectrum of ATOTA.TT in MCH with $13 \mathrm{v} / \mathrm{v} \%$ DCM added largely resembles the spectrum in pure benzene, with only a small solvatochromic shift (ESI, Fig. S6C $\dagger$ ). This supports the conclusion that ATOTA.TT in benzene exists in equilibrium between free dye and ion pair. This agrees with the observation for the other three salts, where the ion pair becomes weaker with increasing size of the counterion.

By subtraction of the spectral component assigned to the free cation it is possible to extrapolate (see Experimental section for details) the spectrum of the pure ATOTA.TT ion pair in benzene (Fig. 6). In this way we are able to obtain the spectra and $\mathrm{S}_{1-}$ $\mathrm{S}_{2}$ splitting for ATOTA.TT both in benzene and $\mathrm{MCH}$ solution (Table 1). The splitting for the $\mathrm{TT}^{-}$salt in benzene is as expected smaller than for the other salts. Comparing the pure ATOTA.TT ion pair in benzene and $\mathrm{MCH}$, we see that the splitting is larger in $\mathrm{MCH}$. We also see that the spectrum in $\mathrm{MCH}$ is switched slightly to the blue compared to that in benzene.

\section{Structure of ATOTA.X contact ion pairs}

In our earlier study, the structure of an ATOTA.PF ${ }_{6}$ contact ion pair was investigated with respect to the relative placement of the anion using semi-empirical methods. ${ }^{17}$ In this work, the equilibrium structures of the contact ion pairs between the $\mathrm{ATOTA}^{+}$cation and the four anions were calculated using DFT structure optimisation. The calculations were performed using 
Table 2 Characteristic distances in the ion pair structures. $\mathrm{X}$ is the central atom of the anion, $\mathrm{N}$ is the closest nitrogen, $\mathrm{O}$ is the oxygen on the edge of $\mathrm{ATOTA}^{+}$facing the anion and $\mathrm{C}$ is the center atom of ATOTA ${ }^{+}$. Radius corresponds to the distance from $\mathrm{C}$ to the most distant atom in the anion

\begin{tabular}{|c|c|c|c|c|}
\hline & $\mathrm{Cl}^{-}$ & $\mathrm{BF}_{4}^{-}$ & $\mathrm{PF}_{6}^{-}$ & $\mathrm{TT}^{-}$ \\
\hline Distance & $\AA$ & $\AA$ & $\AA$ & $\AA$ \\
\hline Cation plane-X & 0.612 & 1.397 & 1.190 & 2.518 \\
\hline$d_{1}: \mathrm{N}-\mathrm{X}$ & 4.424 & 5.512 & 5.873 & 6.299 \\
\hline$d_{2}: \mathrm{O}-\mathrm{X}$ & 3.930 & 3.315 & 3.800 & 6.121 \\
\hline$d_{3}: \mathrm{C}-\mathrm{X}$ & 6.458 & 5.951 & 6.490 & 8.638 \\
\hline Radius & 8.473 & 8.537 & 8.354 & 11.645 \\
\hline
\end{tabular}

four different starting points for each anion, two on the edge and two on the face of the cation. For each salt, all starting geometries converged to a single equilibrium structure, which is different for the different salts. The obtained ion pair structures are shown in Fig. 7 and key distances are given in Table 2.
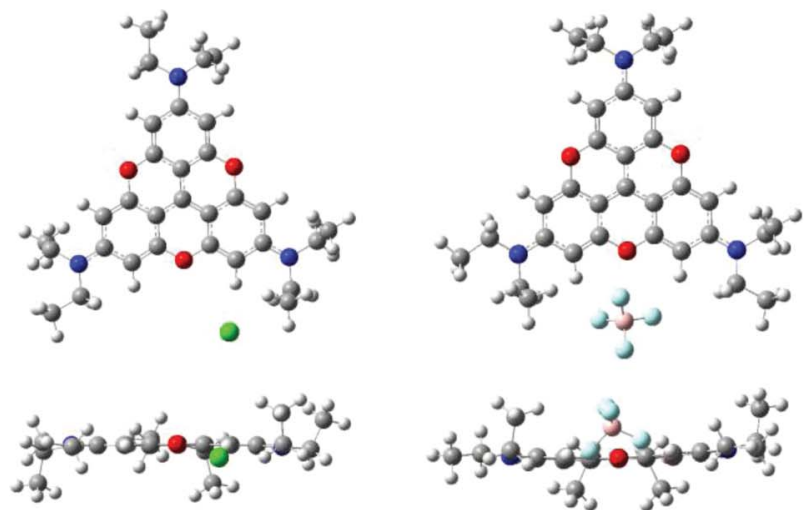
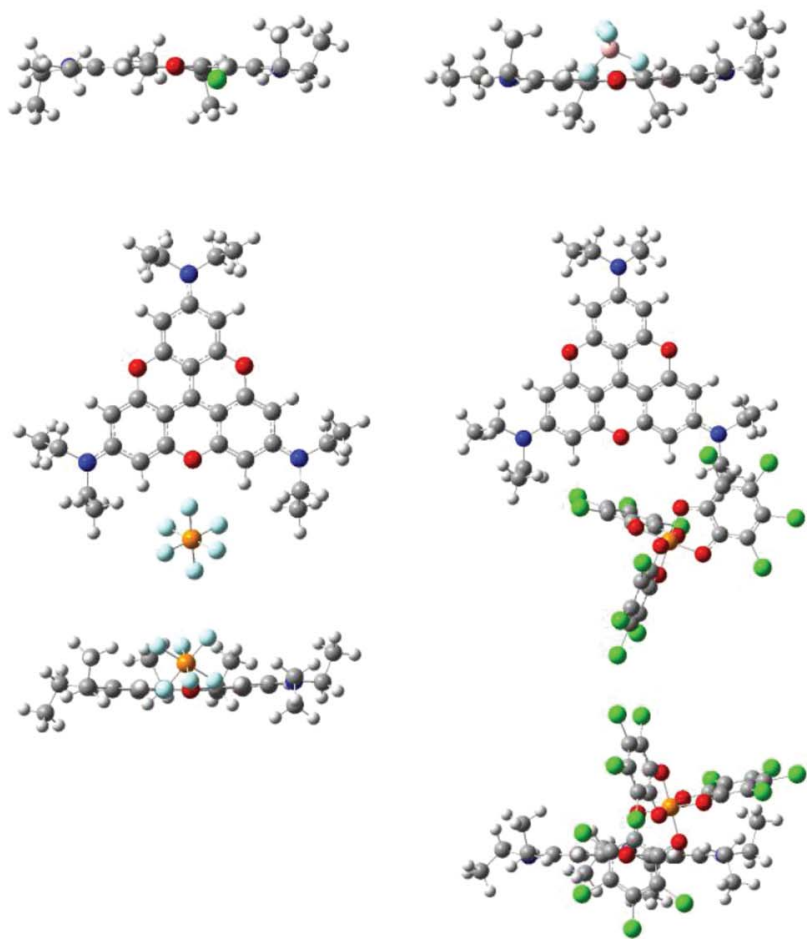

Fig. 7 Calculated structures of contact ion pairs of ethyl substituted ATOTA.X. Carbon (grey), hydrogen (white), boron, (pink), nitrogen(blue), oxygen (red), fluorine (light blue), phosphorous (orange), chloride (green).

Two different ion pair structures are found depending on the anion. One where the anion is placed on one of the symmetry axes of the cation and one where the anion is placed close to one of the three amino groups. The positioning appears to be determined by the closest possible approach. The small $\mathrm{Cl}^{-}$anion can nestle in the nook by the nitrogen, where the fluorinated anions cannot fit. They instead adopt a position close to one of the bridging oxygens. The $\mathrm{TT}^{-}$anion is too large to fit between the amino groups and has to fold around one of them. In all cases the ions are in close contact with the $\mathrm{ATOTA}^{+}$cation. The equilibrium position of the anion is the one that allows for the largest contact area. Thus the complex ions are lifted out of the plane of the cation in order for the cation to be placed closest to the central atom of the anion. This is most evident in case of $\mathrm{TT}^{-}$anion where the arms of the anion wrap around the cation (Fig. 7).

For the symmetric ion pairs $\left(\mathrm{BF}_{4}^{-}\right.$and $\left.\mathrm{PF}_{6}^{-}\right)$there are six equivalent positions for the anions and in the less symmetric cases $\left(\mathrm{Cl}^{-}\right.$and $\left.\mathrm{TT}^{-}\right)$twelve. Previous work showed that there is a barrier for the anions to visit different faces of the cation, but this energy is on the order of $k T$ and the anion will move between these sites at room temperature. ${ }^{17}$ The ion pair structure calculations indicate a significantly broader minimum for the large $\mathrm{TT}^{-}$anion in agreement with the weaker binding observed in the titration experiments.

\section{Electronic structure and excitation energies of ATOTA $^{+}$}

In order to correlate the calculated structures and properties of the ATOTA.X ion pairs with the experimental spectra, electronic transitions in both free $\mathrm{ATOTA}^{+}$cations and the optimised ion pair structures, truncated at ethyl, were calculated.

The structure of the free ATOTA $^{+}$ion was optimised using B3LYP/6-31+G* in vacuum without any constrains. As expected the aromatic core is found to be planar. The excitation energies were calculated using CAM-B3LYP and a variety of basis sets in a dielectric continuum corresponding to benzene $\left(\varepsilon_{\mathrm{r}}=2.27\right.$, $\left.n_{\mathrm{D}}{ }^{2}=2.24\right)$ and $\operatorname{DCM}\left(\varepsilon_{\mathrm{r}}=8.51, n_{\mathrm{D}}{ }^{2}=2.02\right)$, respectively. The data is found to be invariant to basis set size and the results for $6-311++\mathrm{G}^{* *}$ are shown in Table 3 (see ESI for details $\dagger$ ). The electronic excitation energy yields a single double degenerate transition as the lowest allowed transition.

The excitation energy is found to have a negligible splitting of $0.001 \mathrm{eV}$ with equal oscillator strengths. An offset of $\sim 5200$ $\mathrm{cm}^{-1}$ is observed from the experimental data. The nature of the degenerate transition can be described as a movement of electron density from the periphery of the cation to the center. This can be visualised by the HOMO and LUMO orbitals (Table 5), the HOMO is degenerate and the transition moment to the first excited state should in theory be a second rank tensor. It is calculated as two orthogonal transition dipoles, but any normalised linear combination of the two is equally valid. Beside the offset in

Table 3 Calculated electronic properties of free ATOTA ${ }^{+}$

\begin{tabular}{|c|c|c|c|c|c|c|c|}
\hline & \multicolumn{2}{|l|}{$\mathrm{S}_{1}$} & \multirow[b]{2}{*}{$f\left(\mathrm{~S}_{1}\right)$} & \multicolumn{2}{|l|}{$\mathrm{S}_{2}$} & \multirow[b]{2}{*}{$f\left(\mathrm{~S}_{2}\right)$} & \multirow[b]{2}{*}{$\theta_{\mu}{ }^{a}$} \\
\hline & $\mathrm{eV}$ & $\mathrm{cm}^{-1}$ & & $\mathrm{eV}$ & $\mathrm{cm}^{-1}$ & & \\
\hline Vac & 3.31 & 26700 & 0.77 & 3.31 & 26751 & 0.77 & $90.0^{\circ}$ \\
\hline Benzene & 3.27 & 26396 & 0.86 & 3.28 & 26432 & 0.86 & $89.9^{\circ}$ \\
\hline DCM & 3.24 & 26146 & 0.95 & 3.24 & 26167 & 0.95 & $89.9^{\circ}$ \\
\hline
\end{tabular}

${ }^{a}$ Angle between transition moments for $\mathrm{S}_{0} \rightarrow \mathrm{S}_{1}$ and $\mathrm{S}_{0} \rightarrow \mathrm{S}_{2}$, see Fig. 1 . 
Table 4 Calculated and experimental spectroscopic properties of ATOTA.X ion pairs in benzene

\begin{tabular}{|c|c|c|c|c|c|c|c|c|c|c|c|c|}
\hline \multirow[b]{2}{*}{$\mathrm{X}$} & \multicolumn{2}{|l|}{$\mathrm{S}_{1}$} & \multirow[b]{2}{*}{$f\left(\mathrm{~S}_{1}\right)$} & \multicolumn{2}{|c|}{$\mathrm{S}_{1(\exp )}$} & \multicolumn{2}{|l|}{$\mathrm{S}_{2}$} & \multirow[b]{2}{*}{$f\left(\mathrm{~S}_{2}\right)$} & \multicolumn{2}{|c|}{$\mathrm{S}_{2(\exp )}$} & \multirow{2}{*}{$\frac{\mathrm{S}_{1}-\mathrm{S}_{1(\exp )}}{\mathrm{cm}^{-1}}$} & \multirow{2}{*}{$\begin{array}{l}\mathrm{S}_{2}-\mathrm{S}_{2(\exp )} \\
\mathrm{cm}^{-1}\end{array}$} \\
\hline & $\mathrm{eV}$ & $\mathrm{cm}^{-1}$ & & $\mathrm{eV}$ & $\mathrm{cm}^{-1}$ & $\mathrm{eV}$ & $\mathrm{cm}^{-1}$ & & $\mathrm{eV}$ & $\mathrm{cm}^{-1}$ & & \\
\hline none & 3.27 & & 0.86 & - & - & 3.28 & & 0.86 & - & - & - & - \\
\hline $\mathrm{Cl}^{-}$ & 3.13 & 25209 & 0.78 & 2.48 & 20120 & 3.47 & 27970 & 0.73 & 2.74 & 22075 & 5089 & 5895 \\
\hline $\mathrm{BF}_{4}^{-}$ & 3.19 & 25719 & 0.81 & 2.51 & 20284 & 3.42 & 27580 & 0.81 & 2.73 & 22075 & 5437 & 5505 \\
\hline $\mathrm{PF}_{6}^{-}$ & 3.20 & 25807 & 0.83 & 2.52 & 20325 & 3.41 & 27472 & 0.86 & 2.73 & 22026 & 5482 & 5446 \\
\hline $\mathrm{TT}^{-}$ & 3.14 & 25328 & 0.74 & 2.52 & 20243 & 3.34 & 26932 & 0.89 & 2.69 & 21786 & 5085 & 5146 \\
\hline
\end{tabular}

absolute energies the calculations show good agreement with the experimental spectra in polar solvents.

\section{Electronic structure of ATOTA. $X$ ion pairs}

The experimentally observed splitting of the spectra of ATOTA.X ion pairs in solvents of moderate polarity was assigned to tight ion pairs. The nature of this splitting and its structural and electronic origin was investigated further by calculations. Excitation energies were calculated for the four optimised ion pair structures (Fig. 7) using CAM-B3LYP/6-311++ $\mathrm{G}^{* *}$, the results are given in Table 4. The calculations were performed in vacuum and in benzene (see ESI for details $\dagger$ ). Again, an energy shift of $\sim 5100$ $5500 \mathrm{~cm}^{-1}$ between the spectroscopic measurements and the DFT calculations was observed for both $S_{1}$ and $S_{2}$ transitions (Table 4). The calculations show a significant splitting of the first electronic states for all ion pairs. The induced asymmetry is best illustrated by the molecular orbital (MO) plots shown in Table 5. Previous CI calculations showed that the two lowest electronic transitions, are more than $90 \%$ simple HOMO to LUMO, and HOMO-1 to LUMO transitions. ${ }^{17}$ Based on this finding the MOs provide a reasonable visualisation of the $S_{1}$ and $S_{2}$ transitions in the various ion pairs, and can be used to investigate the splitting of the degenerate transition in ATOTA ${ }^{+}$. The low energy transition $\left(\mathrm{S}_{1}\right.$, HOMO to LUMO) is in all ion pairs localised in the diaminoxanthenium chromophore closest to the anion. The $\mathrm{S}_{2}$ transition (HOMO-1 to LUMO) is localised close to orthogonally to the xanthenium type transition in a hemicyanine type chromophore.

HOMO and HOMO- 1 in the ion pairs originate from splitting of the degenerate HOMO in the symmetric ATOTA ${ }^{+}$(Table 5). The symmetry of the frontier orbitals suggests that the exact location of the anion has a huge effect. Symmetrically placed anions $\left(\mathrm{BF}_{4}^{-}, \mathrm{PF}_{6}^{-}\right)$lead to symmetric molecular orbitals, with a clear separation into a xanthenium type HOMO and a hemicyanine type HOMO-1. Anions placed closer to one of the dialkylamino group $\left(\mathrm{Cl}^{-}, \mathrm{TT}^{-}\right)$, lead to unsymmetrical MOs. The influence of the asymmetrically placed anion is clear when the MOs are inspected. The HOMO is primarily localised in the corner of the ATOTA ${ }^{+}$ ion closest to the anion, while HOMO-1 now is localised on two more distant corners, making up a xanthenium like unit opposite to the anion. The smaller ion induces the largest perturbation (Table 5) and we assume this to be due to a larger electric field resulting from the shorter distance between anion and cation. $\mathrm{Cl}^{-}$ is $2 \AA$ closer to $\mathrm{ATOTA}^{+}$than the centre of the $\mathrm{TT}^{-}$ion.

This asymmetry can also be seen in the angle between the calculated transition dipole moments for the transitions to $S_{1}$ and $\mathrm{S}_{2}$, see $\theta_{\mu}$ in Fig. 1 and Table 4 . In the asymmetric ion pairs the two transition dipoles cannot be considered perpendicular as the angle between them approaches $80^{\circ}$ and the symmetry of the system is further reduced. The LUMO is in all cases a fully symmetric MO, with a large coefficient on the central carbon atom (Table 5).

\section{The internal Stark effect}

Table 6 shows that both the experimental and calculated peak splitting is highly dependent on the size of the counterion and decreases as the size of the counterion increases. This supports the hypothesis that the splitting is due to an internal Stark effect i.e. induced by the field generated between the cation and anion.

The calculated peak splitting of the ATOTA.X ion pairs compares well with the experimentally determined value for $\mathrm{BF}_{4}{ }^{-}$, $\mathrm{PF}_{6}{ }^{-}$and $\mathrm{TT}^{-}$. Less so for $\mathrm{Cl}^{-}$where the calculated value is 800 $\mathrm{cm}^{-1}$ larger than the experimental. The most likely explanation for this discrepancy is the applied solvent model that operates with a spherical cavity. In this model the small $\mathrm{Cl}^{-}$ion pair will experience less screening from the solvent. Furthermore any deviation in the calculated cation-anion distance will manifest most strongly in the chloride ion pair due to the strongly nonlinear distance dependence of the electric field (see below).

There is no indication of any specific interaction between the cation and the different anions. Thus, the splitting of the degenerate transition in ATOTA.X seems to be exclusively due to the electric field generated in the ion pair. To further substantiate that the peak splitting is caused by a Stark effect, and not conformational changes of the cation in the ion pair structure, the excitation energies were calculated for the optimised ion pair structures with the anion removed. The result is clearly in favour of the Stark effect hypothesis, as very small peak splittings were found when the anion was removed (see ESI for details $\dagger$ ).

If the peak splitting is a purely electrostatic effect, the distance dependence should adhere to Coulomb's law. This was investigated by varying the distance of $\mathrm{a} \mathrm{BF}_{4}{ }^{-}$ion from $0 \AA$ to $50 \AA$ from the optimised equilibrium position (Fig. 8). As the anion is moved further away, the peak splitting rapidly decreases, converging towards the unperturbed cation. The distance dependence of the peak splitting is in agreement with Coulombs law, falling off as $1 / \mathrm{d}$.

If the peak splitting is due to an internal Stark effect, it should also be induced by an external electric field. Hence, the excitation energies of the free cation were calculated using CAM-B3LYP/6$311++\mathrm{G}^{* *}$, with an external field applied along one axis of the molecule. Fig. 9 shows that the peak splitting can be reproduced using an external field. Thus, it can be concluded that the experimentally observed peak splitting in the ATOTA.X ion pairs is due to the electric field induced by the counterion. Furthermore, only fields applied on the $x$-and $y$-axis of the molecule were found to induce a Stark effect. The results presented in Fig. 9 additionally 
Table 5 Molecular orbital and electron density plots for ATOTA $^{+}$and its ion pairs

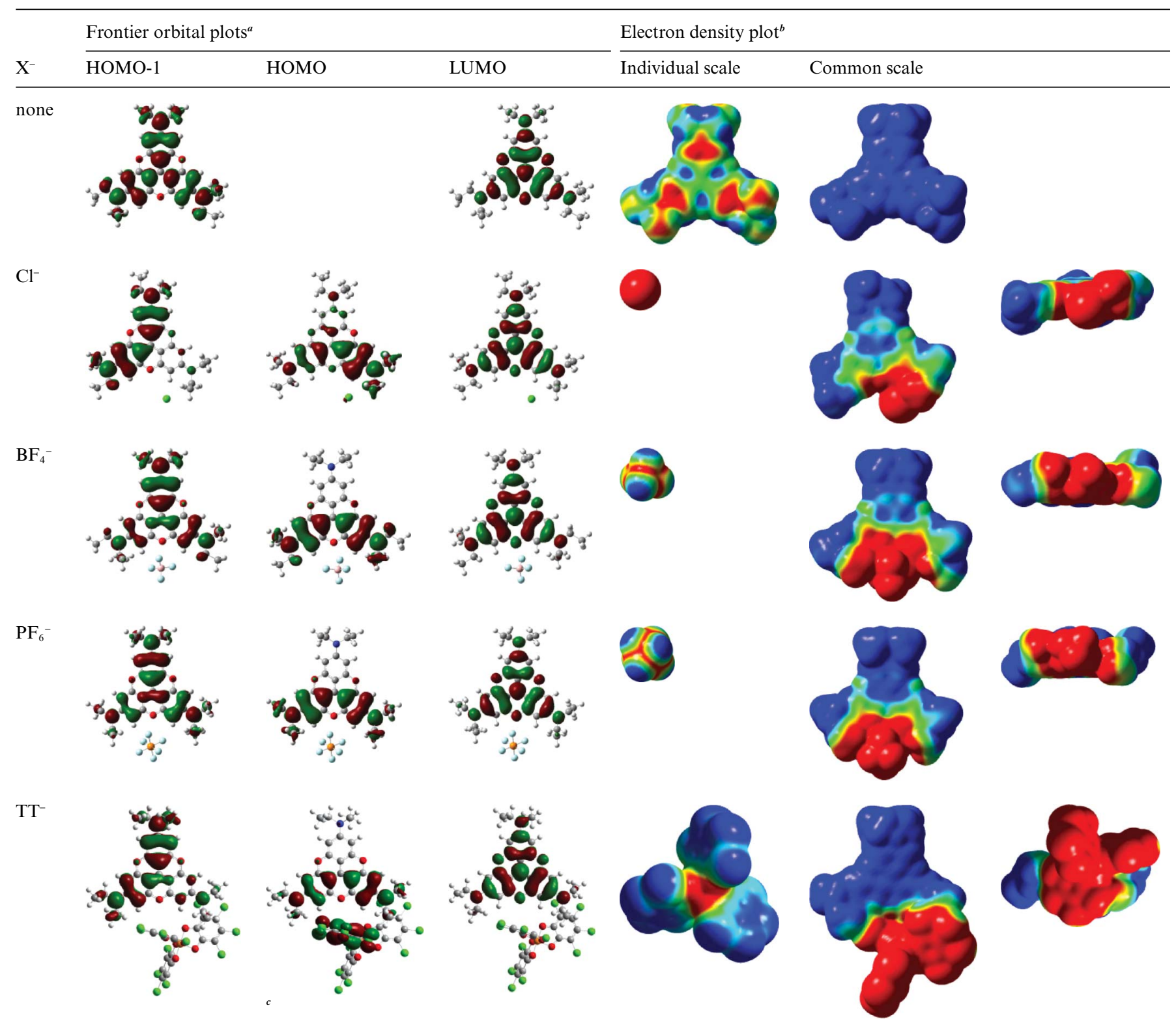

${ }^{a}$ Molecular orbitals calculated using CAM-B3LYP ${ }^{b}$ Relative positive charge: red. Relative negative charge: blue. ${ }^{c}$ The apparent involvement of the orbitals on $\mathrm{TT}^{-}$in this $\mathrm{MO}$ is doubtful and regarded an artifact.

Table 6 Splitting of the electronic transitions in ATOTA.X ion pairs in benzene

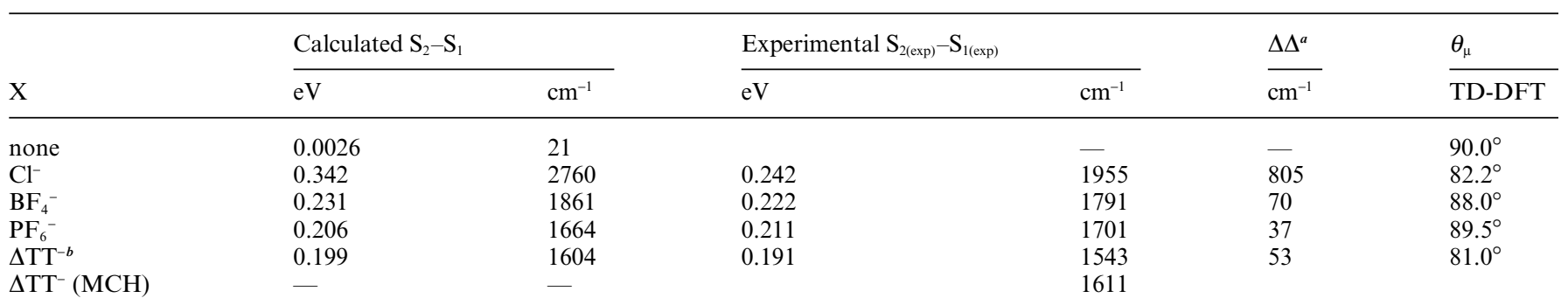

${ }^{a}$ The difference between the experimental and calculated peak splitting. ${ }^{b}$ The $\mathrm{TT}^{-}$ion is calculated with $6-31+\mathrm{G}^{*}$. 


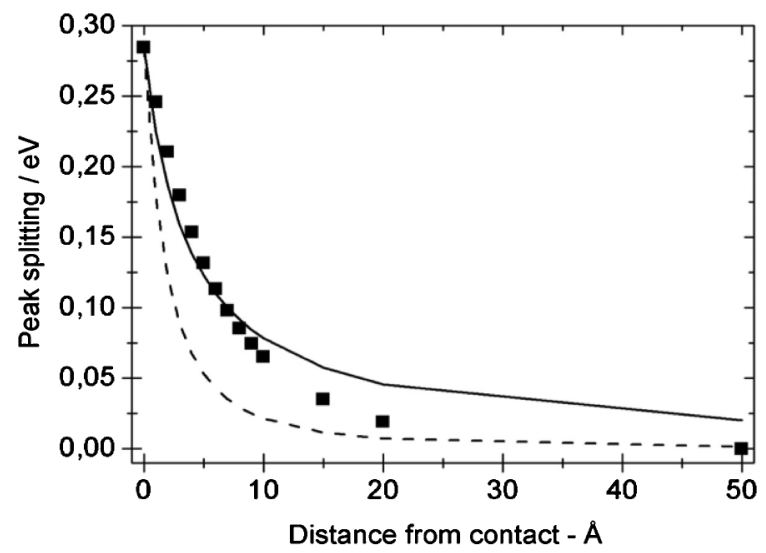

Fig. 8 The calculated distance dependence of the peak splitting of ATOTA $\cdot \mathrm{BF}_{4}(\boldsymbol{\square})$, the distance is given from the equilibrium position. Also plotted is Coulombs law (-) and the distance dependence of charge-dipole interactions (---).

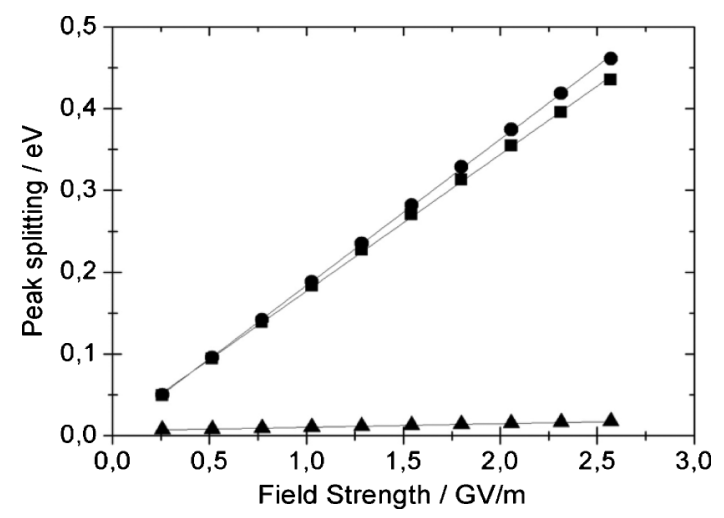

Fig. 9 The calculated peak splitting of the main absorption band of ATOTA $^{+}$as a function of applied field strength. The field was applied along the $x$-axis $(\boldsymbol{\square})$, the $y$-axis $(\mathbf{O})$ and the $z$-axis $(\boldsymbol{\Delta})$ (axes defined in Fig. 1).

shows that the peak splitting is linearly proportional to the field strength, in agreement with what is expected from a Stark effect.

\section{Charge distribution in ATOTA.X ion pairs}

We have previously found that the charge is efficiently delocalised over the entire $\mathrm{ATOTA}^{+}$system with a modicum of positive charge left on the central carbon atom. ${ }^{33}$ In this study, charge densities in ATOTA $^{+}$, the anions and their ion pairs were calculated using B3LYP/6-311++G**. The results are shown in Table 5 on several different scales. The ion pairs are all shown on the same scale in order to compare the degree of anion induced polarisation in the cation. For the free ATOTA $^{+}$cation it is noticed that the positive charge is mainly located on the edge of the triangulenium system in agreement with this being the preferred position for the anions when forming ion pairs. The charge distribution in the anions is included in Table 5. Chloride is a charged sphere. The fluorinated anions and $\mathrm{TT}^{-}$have the negative charge primarily localised on the central atom supporting that this is the centre of the electric field from the anions, that is, the anion can be considered as a point charge placed in the centre of the anion.
For the ion pairs, the perturbation observed in the frontier orbitals, which leads to the observed peak splitting in the absorption spectra, is attributed exclusively to the electric field, i.e. Stark effects. Thus, the polarisation in the electron density distribution of the ion pairs must show similar effects. Table 5 shows that this is indeed the case: the smaller the anion, the closer the charges and the higher the field. The ion pairs of similar symmetry show this. ATOTA $\cdot \mathrm{BF}_{4}$ shows a larger degree of polarisation of the cation than ATOTA. $\mathrm{PF}_{6}$, even though the size difference between the anions in this case is only $0.5 \AA$. In the case of ATOTA.Cl and ATOTA.TT the size difference is $\sim 6 \AA$ and the polarisation of the cation is much smaller in the $\mathrm{TT}^{-}$ion pair.

The electronic effects of the placement of the anion are shown clearly by the electron density plots. Not only is the symmetry of the system affected by the position of the anion, the degree of charge localisation is to a high degree determined by how much of the cation that is in direct contact with the anion. In the $\mathrm{Cl}^{-}$ ion pair the polarisation of the cation is asymmetric, localised at one point of the triangle. In the $\mathrm{BF}_{4}{ }^{-}$and the $\mathrm{PF}_{6}{ }^{-}$ion pairs the polarisation is symmetric over one edge of the triangle, affecting a much larger area of the cation, but to a smaller degree.

\section{ATOTA $^{+}$oriented in a lamellar liquid crystal}

Finally we were interested in experimentally investigating the angle between the two transition moments in the ion pair. To this end we used a lamellar liquid crystal (LLC) based on water, decanol and sodium octanoate that has previously been used to align other chromophores. ${ }^{52}$ In order to increase the orientability of ATOTA ${ }^{+}$ in the amphiphilic lamellar phase we used 2-didecylamino-6,10bis(dimethylamino)-4,8,12-trioxatriangulenium $\left(2^{+}\right)$, a derivative with two n-decyl chains attached to one of the ATOTA ${ }^{+}$corners, and methyl groups at the remaning positions. This compound has previously been studied in Langmuir ${ }^{16}$ and Langmuir-Blodgett films. ${ }^{15}$ It should be noted that the asymmetric substitution pattern of this compound does not affect the photophysical properties of the free ATOTA ${ }^{+}$ion. $^{15}$ The decyl chains prefer the hydrophobic region of the lamellar phase, an effect which is expected to position the other two corners of the ATOTA ${ }^{+}$chromophore close to the anionic heads of the octanoate molecules at the surface of the bilayer (Fig. 10, inset). The isotropic absorption spectrum of this sample (Fig. 10) shows two peaks with a splitting that is similar to that of the $\mathrm{PF}_{6}{ }^{-}$salt in benzene (Table 1; ESI, Fig. S $8 \dagger$ ). The observed splitting suggests that the ATOTA $^{+}$dye in the LLC system is exposed to a local electric field similar to that in the tight ion pair with $\mathrm{PF}_{6}{ }^{-}$in benzene. Interestingly, in the corresponding linear dichorism (LD) spectrum (Fig. 10) the two transitions have opposite signs, the peak at $488 \mathrm{~nm}$ has a negative LD signal, while the $450 \mathrm{~nm}$ peak has a positive LD. In our experimental setup, a negative LD corresponds to a transition oriented along the surface of the amphiphilic bilayer while a positive signal corresponds to a transition parallel to the bilayer normal. According to the calculations above, the red-shifted transition is close to parallel to the side of the triangulenium ion closest to the counterion. That the red-shifted peak in the LD-spectrum is parallel to the bilayer surface is consistent with the $488 \mathrm{~nm}$ transition being closest to the anionic octanoate head groups.

The angle between the two transition moments in the ATOTA ${ }^{+}$ chromophore can be evaluated experimentally by using the 


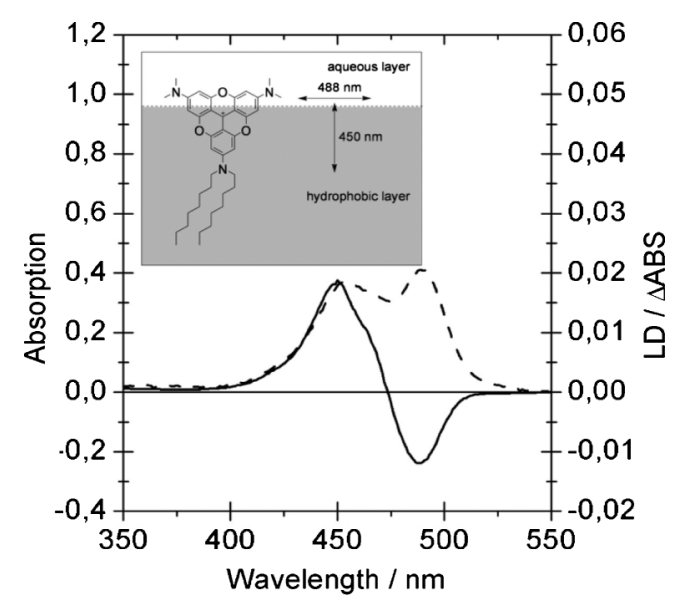

Fig. 10 LD-spectrum (solid line) at a $60^{\circ}$ grazing angle and isotropic absorption (dashed line) of 2-didecylamino-6,10-bis(dimethylamino)-4,8,12-trioxatriangulenium $\left(\mathbf{2}^{+}\right)$oriented in a lamellar liquid crystal. Inset: schematic model of the system, $\left(\mathbf{2}^{+}\right)$is anchored into the lamellar bilayer (shadowed) by two decyl chains. The arrows indicate the direction of the two transitions. The dye can rotate freely around the surface normal. The dye is inserted arbitrarily deep into the bilayer. The dashed line corresponds to the heads of the negatively charged octanoate molecules.

reduced $\mathrm{LD}\left(\mathrm{LD}^{\mathrm{r}}\right)$, the $\mathrm{LD}$ divided by the isotropic absorption ( $\left.A_{\text {iso }}\right)$ according to eqn (2):.$^{53}$

$$
\mathrm{LD}^{\mathrm{r}}=\mathrm{LD} / A_{\text {iso }}=3 S\left(3 \cos ^{2} \alpha_{i}-1\right) / 2
$$

(see ESI for details on the calculations). The orientation factor $S$ describes the average alignment of $\mathbf{2}^{+}$with respect to the normal to the quartz surfaces that are used to confine the lamellar phase. The $S$-value is identical for the two transitions, which differ in the angle $\alpha_{i}$ that describes their respective orientation in the $\mathrm{ATOTA}^{+}$ framework. With the chosen coordinate system (ESI, Fig. S9 $\dagger$ ) and assuming $\alpha_{488}=90^{\circ}$ for the $488 \mathrm{~nm}$ transition, eqn (2) gives an $S$-value that can be used to calculate $\alpha$ for the $450 \mathrm{~nm}$ transition. Doing so we obtain $\alpha_{450}=15^{\circ}$ (for details see the ESI), close enough to the predicted value of $0^{\circ}$ to confirm that the two transitions are orthogonal to each other within experimental error.

The LD-study provides clear evidence that the two transitions in the ion pair spectra are nearly perpendicular oriented in the molecule and that the $S_{1}$ transition is polarized along the axis connecting the two donor nitrogen groups closest to the anions. Both findings are in excellent agreement with the theoretical models.

\section{Summary and conclusion}

The electronic properties of ion pairs of 2,6,10tris(dioctylamino)trioxatriangulenium $\mathrm{Cl}^{-}, \mathrm{BF}_{4}^{-}, \mathrm{PF}_{6}^{-}$and $\mathrm{TT}^{-}$have been investigated using spectroscopy and quantum chemical calculations. The absorption spectrum of free 2,6,10tris(dioctylamino)trioxatriangulenium contains one degenerate electronic transition in the visible, which upon ion pair formation is split into two bands. The separation of the two bands is determined by the size of the anion. By a combination of direct and indirect theoretical calculations, the splitting is shown to be caused by an internal Stark effect in the ion pairs. The electric field generated between the cation and anion in the contact ion pairs is on the order of $1 \mathrm{GV} \mathrm{m}^{-1}$. By orienting an amphiphilic ATOTA $^{+}$dye in a negatively charged lipid bilayer it was possible to resolve orientation of the two transitions in the ion pair spectrum, providing a strong support for our interpretation of the calculations and spectroscopy on isotropic solutions. We conclude that the splitting of the electronic states in ATOTA $^{+}$ dyes in non-polar solvents can be fully explained by an internal Stark effect in the formed ion pairs. This conclusion is most likely also true for other symmetric triaryl methylium dyes.

\section{Acknowledgements}

This work was supported by the Danish National Research Foundation under the Danish-Chinese Centre for Self-Assembled Molecular Electronic Nanosystems. FW acknowledges funding from the Knut and Alice Wallenberg Foundaiton and the Chalmers Area of Advance in Nanoscience and Nanotechnology. TJS thanks the Danish Council for Independent Research | Technology and Production Sciences (grant 10-093546) for financial support. KVM, thanks the Danish Center for Scientific Computing, The Danish Council for Independent Research I Natural Sciences, and the Villum Kann Rasmussen Foundation for financial support.

\section{Notes and references}

1 I. Johnson, Fluorescent probes for living cells, Histochem. J., 1998, 30, $123-140$.

2 J. W. Lichtman and J. A. Conchello, Fluorescence microscopy, Nat. Methods, 2005, 2, 910-919.

3 B. N. G. Giepmans, S. R. Adams, M. H. Ellisman and R. Y. Tsien, Review - the fluorescent toolbox for assessing protein location and function, Science, 2006, 312, 217-224.

4 A. M. Smith, M. C. Mancini and S. Nie, Bioimaging: Second window for in vivo imaging, Nat. Nanotechnol., 2009, 4, 710-711.

5 V. Ntziachristos, Going deeper than microscopy: The optical imaging frontier in biology, Nat. Methods, 2010, 7, 603-614.

6 S. R. Adams, A. T. Harootunian, Y. J. Buechler, S. S. Taylor and R. Y. Tsien, Fluorescence ratio imaging of cyclic-amp in single cells, Nature, 1991, 349, 694-697.

7 J. W. Bell and N. M. Hext, Supramolecular optical chemosensors for organic analytes, Chem. Soc. Rev., 2004, 33, 589-598.

8 S. M. Borisov and O. S. Wolfbeis, Optical biosensors, Chem. Rev., 2008, 108, 423-461.

9 C. McDonagh, C. S. Burke and B. D. MacCraith, Optical chemical sensors, Chem. Rev., 2008, 108, 400-422.

10 A. Mishra, R. K. Behera, P. K. Behera, B. K. Mishra and G. B. Behera, Cyanines during the 1990s: A review, Chem. Rev., 2000, 100, 1973-2012.

11 B. Baisch, D. Raffa, U. Jung, O. M. Magnussen, C. Nicolas, J. Lacour, J. Kubitschke and R. Herges, Mounting freestanding molecular functions onto surfaces: The platform approach, J. Am. Chem. Soc., 2009, 131, $442-443$.

12 S. K. Narasimhan, D. J. Kerwood, L. Wu, J. Li, R. Lombardi, T. B. Freedman and Y.-Y. Luk, Induced folding by chiral nonplanar aromatics, J. Org. Chem., 2009, 74, 7023-7033.

13 Y. Haketa, S. Sasaki, N. Ohta, H. Masunaga, H. Ogawa, N. Mizuno, F. Araoka, H. Takezoe and H. Maeda, Oriented salts: Dimensioncontrolled charge-by-charge assemblies from planar receptor-anion complexes, Angew. Chem., Int. Ed., 2010, 49, 10079-10083.

14 S. Kuhn, B. Baisch, U. Jung, T. Johannsen, J. Kubitschke, R. Herges and O. Magnussen, Self-assembly of triazatriangulenium-based functional adlayers on au(111) surfaces, Phys. Chem. Chem. Phys., 2010, 12, 44814487.

15 J. B. Simonsen, F. Westerlund, D. W. Breiby, N. Harrit and B. W. Laursen, Columnar self-assembly and alignment of planar carbenium ions in Langmuir-Blodgett films, Langmuir, 2011, 27, 792-799. 
16 J. B. Simonsen, K. Kjaer, P. Howes, K. Nørgaard, T. Bjørnholm, N. Harrit and B. W. Laursen, Close columnar packing of triangulenium ions in langmuir films, Langmuir, 2009, 25, 3584-3592.

17 B. W. Laursen, J. Reynisson, K. V. Mikkelsen, K. Bechgaard and N. Harrit, 2,6,10-tris(dialkylamino)trioxatriangulenium salts: A new promising fluorophore. Ion-pair formation and aggregation in nonpolar solvents, Photochem. Photobiol. Sci., 2005, 4, 568-576.

18 L. M. Lewis and G. L. Indig, Solvent effects on the spectroscopic properties of triarylmethane dyes, Dyes Pigm., 2000, 46, 145-154.

19 D. F. Duxbury, The photochemistry and photophysics of triphenylmethane dyes in solid and liquid-media, Chem. Rev., 1993, 93, 381-433.

20 J. E. Selwyn and J. I. Steinfeld, Aggregation of equilibriums of xanthene dyes, J. Phys. Chem., 1972, 76, 762-774.

21 G. U. Bublitz and S. G. Boxer, Stark spectroscopy: Applications in chemistry, biology, and materials science, Annu. Rev. Phys. Chem., 1997, 48, 213-242.

22 R. P. Haugland, Handbook of fluorescent probes and research chemicals, Molecular Probes, Eugene, Oregon, 1996.

23 G. N. Lewis, T. T. Magel and D. Lipkin, Isomers of crystal violet ion. Their absorption and re-emission of light, J. Am. Chem. Soc., 1942, 64, 1774-1782.

24 H. B. Lueck, J. L. McHale and W. D. Edwards, Symmetry-breaking solvent effects on the electronic structure and spectra of a series of triphenylmethane dyes, J. Am. Chem. Soc., 1992, 114, 2342-2348.

25 F. C. Adam and W. T. Simpson, Electronic spectrum of 4, 4'-bisdimethylamino fuchsone and related triphenylmethane dyes, J. Mol. Spectrosc., 1959, 3, 363-380.

26 C. S. Oliveira, K. P. Branco, M. S. Baptista and G. L. Indig, Solvent and concentration effects on the visible spectra of tri-para-dialkylaminosubstituted triarylmethane dyes in liquid solutions, Spectrochim. Acta, Part A, 2002, 58, 2971-2982.

27 J. Korppi-Tommola and R. W. Yip, Solvent effects on the visible absorption spectrum of crystal violet, Can. J. Chem., 1981, 59, 191194.

28 F. Feichtmayr and J. Schlag, Influence of solvent and concentration on the spectra of triphenylmethane dyes, Berichte der Bunsen-Gesellschaft, 1964, 68, 95-102.

29 B. W. Laursen, F. C. Krebs, M. F. Nielsen, K. Bechgaard, J. B. Christensen and N. Harrit, 2,6,10-tris(dialkylamino)trioxatriangulenium ions. Synthesis, structure, and properties of exceptionally stable carbenium ions, J. Am. Chem. Soc., 1998, 120, 12255-12263.

30 S. Dileesh and K. R. Gopidas, Photophysical and electron transfer studies of a stable carbocation, Chem. Phys. Lett., 2000, 330, 397-402.

31 S. Dileesh and K. R. Gopidas, Photoinduced electron transfer in azatriangulenium salts, J. Photochem. Photobiol., A, 2004, 162, 115120.

32 A. Pothukuchy, C. L. Mazzitelli, M. L. Rodriguez, B. Tuesuwan, M. Salazar, J. S. Brodbelt and S. M. Kerwin, Duplex and quadruplex DNA binding and photocleavage by trioxatriangulenium lone, Biochemistry, 2005, 44, 2163-2172.

33 B. W. Laursen and T. J. Sørensen, Synthesis of super stable triangulenium dye, J. Org. Chem., 2009, 74, 3183-3185.

34 T. J. Sørensen, B. W. Laursen, R. Luchowski, T. Shtoyko, I. Akopova, Z. Gryczynski and I. Gryczynski, Enhanced fluorescence emission of meadota (+) by self-assembled silver nanoparticles on a gold film, Chem. Phys. Lett., 2009, 476, 46-50.

35 F. Westerlund, C. B. Hildebrandt, T. J. Sørensen and B. W. Laursen, Trihydroxytrioxatriangulene - an extended fluorescein and a ratiometric ph sensor, Chem.-Eur. J., 2010, 16, 2992-2996.
36 T. J. Sørensen and B. W. Laursen, Synthesis and optical properties of trioxatriangulenium dyes with one and two peripheral amino substituents, J. Org. Chem., 2010, 75, 6182-6190.

37 B. W. Laursen and F. C. Krebs, Synthesis of a triazatriangulenium salt, Angew. Chem., Int. Ed., 2000, 39, 3432-3434.

38 B. W. Laursen and F. C. Krebs, Synthesis, structure, and properties of azatriangulenium salts, Chem.-Eur. J., 2001, 7, 1773-1783.

39 F. Favarger, C. Goujon-Ginglinger, D. Monchaud and J. Lacour, Large-scale synthesis and resolution of trisphat [tris(tetrachlorobenzenediolato) phosphate(v)] anion, J. Org. Chem., 2004, 69, 8521-8524.

40 A. D. Becke, Density-functional exchange-energy approximation with correct asymptotic-behavior, Phys. Rev. A: At., Mol., Opt. Phys., 1988, 38, 3098-3100.

41 C. T. Lee, W. T. Yang and R. G. Parr, Development of the colle-salvetti correlation-energy formula into a functional of the electron-density, Phys. Rev. B, 1988, 37, 785-789.

42 A. D. Becke, Density-functional thermochemistry .3. The role of exact exchange, J. Chem. Phys., 1993, 98, 5648-5652.

43 P. J. Stephens, F. J. Devlin, C. F. Chabalowski and M. J. Frisch, Ab initio calculation of vibrational absorption and circular dichroism spectra using density functional force fields, J. Phys. Chem., 1994, 98, 11623 11627.

44 T. Yanai, D. P. Tew and N. C. Handy, A new hybrid exchangecorrelation functional using the coulomb-attenuating method (camb3lyp), Chem. Phys. Lett., 2004, 393, 51-57.

45 A. Dreuw, J. L. Weisman and M. Head-Gordon, Long-range chargetransfer excited states in time-dependent density functional theory require non-local exchange, J. Chem. Phys., 2003, 119, 29432946.

46 Y. Tawada, T. Tsuneda, S. Yanagisawa, T. Yanai and K. Hirao, A longrange-corrected time-dependent density functional theory, J. Chem. Phys., 2004, 120, 8425-8433.

47 M. J. G. Peach, P. Benfield, T. Helgaker and D. J. Tozer, Excitation energies in density functional theory: An evaluation and a diagnostic test, J. Chem. Phys., 2008, 128, 044118.

48 DALTON, a molecular electronic structure program, see http://www.kjemi.uio.no/software/dalton/dalton.html, 2005, Release 2.0.

49 J. Lacour, G. Bernardinelli, V. Russell and I. Dance, Crystal packing interpretation of the association of chiral threefold propeller ions: Trisphat anion with a triarylcarbenium cation, CrystEngComm, 2002, 4, 165-170.

50 R. Friman, I. Danielsson and P. Stenius, Lamellar mesophase with high contents of water - X-ray-investigations of the sodium octanoate decanol water system, J. Colloid Interface Sci., 1982, 86, 501514.

51 K. Fontell, L. Mandell, H. Lehtinen and P. Ekwall, 3-component system sodium caprylate - decanol - water .3. Structure of mesophases at 20 degrees c, Acta Polytechnica Scandinavica-Chemistry Including Metallurgy Series, 1968, 1-56.

52 B. Nordén, G. Lindblom and I. Jonas, Linear dichroism spectroscopy as a tool for studying molecular orientation in model membrane systems, J. Phys. Chem., 1977, 81, 2086-2093.

53 B. Nordén, A. Rodger and T. R. Dafforn, Linear dichroism and circular dichroism - a textbook on polarized-light spectroscopy, RSC Publishing, Cambridge, UK, 2010.

54 Y. Marcus and G. Hefter, Ion pairing, Chem. Rev., 2006, 106, 45854621. 